\title{
Bayesian Inference of Reliability Growth- Oriented Weibull Distribution for Multiple Mechanical Stages Systems
}

\author{
M. Nadjafi ${ }^{*}$, P. Gholami ${ }^{2}$ \\ 1. Aerospace Research Institute (Ministry of Science, Research and Technology), Tehran, Iran \\ 2. Department of Aerospace Engineering, Sharif University of Technology, Tehran, Iran
}

\begin{abstract}
The Duane and Crow-AMSAA reliability growth model has been traditionally used to model systems and products undergoing development testing. The Non-Homogeneous Poisson Process (NHPP) with a power intensity law has been often used as a model for describing the failure pattern of the repairable systems and the maximum likelihood (ML) estimates are used to calculate the unknown parameters widely. This study proposes the statistical analysis method of different stages and different level data based on Bayes analysis techniques. To this end, the Bayesian reliability growth model of multiple stages is coupled with the Weibull distribution product. By using the unique properties of the assumed prior distributions, the moments of the posterior distribution of the failure rate at various stages during a development test can be found. In this paper, it is assumed that the scale parameter has a Gamma prior density function, and the growth parameter has a Uniform prior distribution. Monte Carlo simulations are used to compute the Bayes estimates. Finally, the results obtained from the proposed method by implementing it on an application example are compared with Crow-AMSAA data and show that the proposed model has higher accuracy than the existing traditional methods.
\end{abstract}

Keyword: Reliability Growth; Non-Homogeneous Poisson Process (NHPP); Bayes Analysis; Weibull Distribution; Monte Carlo Simulations

\section{Nomenclature}

$\begin{array}{ll}a & \text { Parameter of Gamma Distribution } \\ b & \text { Parameter of Gamma Distribution } \\ L & \text { Likelihood function } \\ m & \text { Number of stages } \\ n & \text { Number of failures } \\ N & \text { Cumulative number of failures } \\ R & \text { Reliability } \\ T & \text { Mission time } \\ \lambda & \text { Scale parameter } \\ \beta & \text { Shape parameter } \\ \varphi & \text { Density function } \\ \Gamma & \text { Gamma function } \\ C L & \text { Confides level } \\ \text { ML } & \text { Maximum likelihood } \\ \text { MTBF } & \text { mean time between failure } \\ \text { MCMC } & \text { Markov Chain Monte Carlo } \\ \text { NHPP } & \text { Nonhomogeneous Poisson Process }\end{array}$

\footnotetext{
* Corresponding Author Email: m.nadjafi@ari.ac.ir
} 
statistical extension became what is known as the CrowAMSAA (NHPP) model. The Crow-AMSAA model is designed for tracking the reliability within a test phase. A development-testing program may consist of several separate test phases. In recent years, there has been much effort to develop the Crow-AMSAA model and create better models for mechanical systems [3-6]. Wang et al. [7] studied a reliability growth testing allocation problem to series-parallel systems that consider parameter uncertainty in the Crow-AMSAA models. They assumed the parameters of the model to be known as uncertain-but-bounded values. Lee and his colleagues [8] used the Crow-AMSAA model to analyze the reliability growth of multiple launch rocket components by using test data obtained from the development phase. Recently, the Crow-AMSAA model was developed by Nadjafi and Gholami [9] using the normal distribution and maximum likelihood (ML) estimate and proved its effectiveness on the data of an aerospace system. The main purpose of this study is to develop a model for the growth of reliability with a normal distribution based on the NHPP. Also, to evaluate the reliability model with the given failure data, the maximum likelihood estimation technique was used to estimate the effective parameter in reliability growth. Although current study proposes the statistical analysis method of different stages and different level data based on Bayes analysis techniques and gave the Bayesian reliability growth model of multiple stages Weibull distribution product.

Given that the reliability growth models predict the reliability at different stages of the developmental process, depending on the nature of a process, various models can be used to estimate the desired parameters. Reliability growth models can be deterministic or probabilistic. They can be based on the classical or the Bayes estimation method. The classical approach to reliability growth estimation normally is in the form of a mathematical formula with one or more parameters to be estimated from the data collected at each stage of development. Estimates of these unknown parameters are usually obtained using Maximum Likelihood (ML) or least-squares estimators and are anticipated to reflect the characteristics of the system.

When experimental data are limited, as they often are inexpensive or time-consuming testing and development percentage, it may be desirable to use prior information in conjunction with the data to estimate unknown parameters. Hence, Bayes methods may be desirable as they allow prior information to be incorporated into the inferential procedure. The Bayes approach to reliability growth estimation usually begins with an assumption of a prior distribution. The estimates of the prior distribution parameters are updated as samples from each development stage are collected.
Several authors have proposed Bayes reliability growth models. The original work by Smith [10] developed a Bayes algorithm to estimate the reliability of a system during the development period. Fard \& Dietrich [11] used Bayesian approaches for analyzing reliability growth that proposed a Bayesian analysis of the problem where failure causes are lumped into one category and a jointly ordered uniform prior is assumed for the reliability of the system at each stage. Robinson and Dietrich [12] propose a nonparametric reliability growth model based on Bayes analysis techniques.

$\mathrm{Lu}$ and his colleagues [13] by considering the system debugging test phase as a Non-Homogeneous Poisson Process (NHPP) and the reliability demonstration test phase as a homogeneous Poisson Process established a joint likelihood function between the failure intensity of these two steps. The Bayesian method was used to obtain the upper limit of the failure intensity. A degradation model with a random failure threshold is proposed by Huang et al. [14] for the evaluation of the reliability of the multi-stage system by the Bayesian approach. Ming et al. [15] investigated Bayesian reliability growth models of the mechanical systems using new Dirichlet prior distribution when the sample of the system is small.

Ruiz and his colleagues [16] developed a Bayesian framework to analyze accelerated life testing data in reliability growth. In this study, it is assumed that the failure modes of components of the system have multiple competing failure modes and the time to failure of each failure mode follows a Weibull distribution. Park et al. [17] used the Bayesian method to estimate the parameters of the reliability growth model when the number of sample data for the fault information is small and showed that the estimation accuracy of the Bayesian method is more accurate than that of Maximum Likelihood (ML) Estimation. Using multi-stage data in the product development test, Wang et al. [18] proposed a scaling factor method to calculate reliability growth and then used Bayesian posterior estimation to determine reliability parameters using the Markov Chain Monte Carlo (MCMC) sampling method.

In this paper, the reliability growth of a system with $m$ development process stages is studied. It is assumed that failures are removed after each testing phase. In this regard, the moments of the marginal distribution at various stages during a development test are used and the proposed model is compared with the CrowAMSAA model based on relative and mean square prediction errors. The quality and reliability index of the considered system is not constant due to the removal of failures on each state. Therefore, the assessment is not accurate and effective when using the traditional reliability growth model. Thus, according to these problems, in this paper, Bayes estimates are derived for 
analyzing reliability growth with the assumption of an underlying distribution to describe the time to failure during any stages. In Bayesian statistics, the posterior parameter distribution is used for calculating parameter point estimates, to construct interval estimates or to predict future process values and provide the multistage Bayes reliability growth model of Weibull distribution. It should be assumed some prior distributions of the unknown parameters. Therefore, in this study, it is assumed that the scale parameter has a Gamma prior density function, and the growth parameter has a Uniform prior distribution. In the following, Monte Carlo techniques are used to compute the Bayes estimates. Then, the results of Bayes estimates have been compared with the traditional reliability growth model by a worked example.

\section{Modelling framework Equipment}

Depending on the characteristics of the system, some strategies and a number of phases could be created in the testing program. Indeed, the test program is applied to catch errors and defect in each phase of the defined lifecycle of the system. So, the number of test phases is determined in such a way that all the predicted and also the unpredicted failures in terms of project management are removed until the requirements of the user are satisfied. The reliability growth testing program provides opportunities to identify the weaknesses and failure modes in the design and manufacturing process. So, management makes decisions regarding the management strategy in each stage of the test program to correct problems or not correct problems and the effectiveness of the corrective actions. Assuming the reliability growth process of the product includes $m$ stages and each stage is independent. The number of failures in each stage is $n_{1}, n_{2} \ldots, n_{m}$. Product time to failure is Weibull distribution in the $i$ th stage, its hazard function is $\lambda(t)=\lambda \beta t^{\beta-1}$, if the mission time is $T$, then the product reliability of the $i$ th stage is:

$R_{k}=R_{k}\left(T_{k} \geq T\right)=\exp \left(-\lambda t^{\beta}\right)$

Here, $\lambda$ is scale parameter and $\beta$ is growth parameter. It is worth mentioning that the major advantages of using Weibull analysis are that it can be used for analyzing lifetimes with very small samples that can cover all regions of the life cycle (includes: increasing failure rate-IFR, constant failure rate CFR, and decreasing failure rate-DFR). It also produces an easy-to-understand plot. After each stage product reliability continuously raises because of removing defects, thus

$0 \leq R_{1} \leq R_{2} \leq \ldots \leq R_{m} \leq 1$

Letting $N(t)$ be the cumulative number of failures observed by time $T$, therefore $N(t)$ can be modeled as a Non-Homogeneous Poisson process (NHPP), i.e., as a Poisson process with a time-dependent failure rate
$P\{N(T)=k\}=\frac{[\omega(T)]^{k} e^{-\omega(T)}}{k !} ; k=0,1,2, \ldots$

Where $\omega(t)=\lambda T^{\beta}$, that if $\beta>1$ implying wear-out, $\beta<1$ implying growth and $\beta=1$ implying constant mean time between failure (MTBF).

Bayes' rule provides the framework for combining prior information with sample data. In this reference, we apply Bayes' rule for combining prior information on the assumed distribution's parameters with sample data in order to make inferences based on the model. The prior knowledge about the parameters is expressed in terms of $\varphi(\lambda)$ and $\varphi(\beta)$ called the prior distribution. The posterior distribution of $\lambda$ and $\beta$ given the sample data, using Bayes' rule, provides the updated information about the parameters $\lambda$ and $\beta$. This is expressed with the following posterior pdf

$$
f(\lambda, \beta \mid D)=\frac{L(\lambda, \beta) \varphi(\lambda) \varphi(\beta)}{\iint_{\Omega} L(\lambda, \beta) \varphi(\lambda) \varphi(\beta) d \lambda d \beta}
$$

Where $\lambda$ and $\beta$ is a vector of the parameters of the chosen distribution, $\Omega$ is the range of $\lambda$ and $\beta, L(\lambda, \beta)$ is the likelihood function based on the chosen distribution and data, $\varphi(\lambda)$ and $\varphi(\beta)$ is the prior distribution for each of the parameters. In other words, a distribution (the posterior pdf) is obtained, rather than a point estimate as in classical statistics. Therefore, if a point estimate needs to be reported, a point of the posterior pdf needs to be calculated. Typical points of the posterior distribution used are the mean (expected value).

It is desired that the prior distribution on the failure rate at each stage be consistent in terms of information available, and at the same time not be a computational burden. In this model $\beta$ is assumed to follow prior distribution with the density function $\varphi(\beta)=1 / \beta$. The prior distribution of $\lambda$, denoted as $\varphi(\lambda)$, can be assumed Gamma distribution with density function $\varphi(\lambda)=\left[b^{\gamma} \lambda^{\gamma-1} / \Gamma(a)\right] \exp (-\lambda b)$, where $\Gamma(\cdot)$ is gamma function, while $a$ and $b$ represent gamma parameters.

The joint prior distribution on the successive failure rates is

$$
g\left(\lambda_{k}, \beta_{k}\right)=\frac{\prod_{i=1}^{m} \varphi\left(\lambda_{i} \mid a, b\right) \varphi\left(\beta_{i}\right)}{\iint \prod_{\Omega}^{m} \varphi\left(\lambda_{i}\right) \varphi\left(\beta_{i}\right) d \lambda d \beta}
$$

From Bayes theorem, it is known that the joint posterior pdf of $\lambda$ and $\beta$ is

$$
f\left(\lambda_{i}, \beta_{i}\right)=\frac{\prod_{i=1}^{m} h\left(\lambda_{i}, \beta_{i} \mid n_{i}\right) \varphi\left(\lambda_{i} \mid a_{i}, b_{i}\right) \varphi\left(\beta_{i}\right)}{\iint_{\Omega} \prod_{i=1}^{m} h\left(\lambda_{i}, \beta_{i} \mid n_{i}\right) \varphi\left(\lambda_{i} \mid a_{i}, b_{i}\right) \varphi\left(\beta_{i}\right) d \lambda d \beta}
$$

Where, 
$h\left(\lambda_{i}, \beta_{i} \mid n_{i}\right)=\frac{[\omega(T)]^{n_{i}} e^{-\omega(T)}}{n_{i} !}$

Implementation of these priors and solving for the marginal distribution, $f\left(\lambda_{i}, \beta_{i} \mid D\right)$, still remains a difficult problem. However, it is relatively easy to find the moments of this distribution and thus an approximation to the distribution can be found to any desired accuracy.

The expected value of $\beta$ is obtained by

$$
\begin{aligned}
E\left(\beta_{i} \mid D\right)= & \iint_{\Omega} \beta_{i} \cdot f\left(\lambda_{i}, \beta_{i} \mid D\right) d \lambda d \beta \\
= & \frac{\int 00 \prod_{i=1}^{\infty} \lambda_{i}^{n_{i}+a_{i}-1} \exp \left(-\lambda_{i}\left(T_{i}+b_{i}\right)\right) d \lambda d \beta}{\iint_{0}^{\infty} \int_{0}^{m}\left[\lambda_{i=1}^{m}\left[\lambda_{i}{ }^{{ }_{i}+a_{i}-1} \exp \left(-\lambda_{i}\left(T_{i}+b_{i}\right)\right) / \beta_{i}\right] d \lambda d \beta\right.}
\end{aligned}
$$

Similarly, the expected value of $\lambda$ is obtained by

$$
\begin{aligned}
E\left(\lambda_{i} \mid D\right)= & \iint_{\Omega} \lambda \cdot f\left(\lambda_{i}, \beta_{i} \mid D\right) d \lambda d \beta \\
= & \frac{\int_{0}^{\infty} \int_{0}^{\infty} \prod_{i=1}^{m}\left[\lambda_{i}^{n_{i}+a_{i}} \exp \left(-\lambda_{i}\left(T_{i}+b_{i}\right)\right) / \beta_{i}\right] d \lambda d \beta}{\iint_{0}^{\infty} \int_{0}^{m} \prod_{i=1}^{m}\left[\lambda_{i}^{n_{i}+a_{i}-1} \exp \left(-\lambda_{i}\left(T_{i}+b_{i}\right)\right) / \beta_{i}\right] d \lambda d \beta}
\end{aligned}
$$

We now have the distribution of $\lambda$ and $\beta$, hence we can now make statistical inferences on this parameter, such as calculating probabilities. Specifically, the probability that $\lambda$ and $\beta$ fall within a specified value $p\left(\beta_{L} \leq \beta \leq \beta_{U}, \lambda_{L} \leq \lambda \leq \lambda_{U}\right)$ can be obtained by integrating the posterior probability density function (pdf), or

$$
\begin{aligned}
C L & =p\left(\beta_{L} \leq \beta \leq \beta_{U}, \lambda_{L} \leq \lambda \leq \lambda_{U}\right) \\
& =\int_{\lambda_{L}}^{\lambda_{U}} \int_{\beta_{L}}^{\beta_{U}} f\left(\lambda_{i}, \beta_{i} \mid D\right) d \lambda d \beta
\end{aligned}
$$

Which is equivalent to:

$$
\begin{aligned}
& (1+C L) / 2=\int_{0}^{\lambda_{U}} \int_{0}^{\beta_{U}} f\left(\lambda_{i}, \beta_{i} \mid D\right) d \lambda d \beta \\
& (1-C L) / 2=\int_{0}^{\lambda_{L}} \int_{0}^{\beta_{L}} f\left(\lambda_{i}, \beta_{i} \mid D\right) d \lambda d \beta
\end{aligned}
$$

Where $C L$ is the confidence level. The above equations can be generalized for any distribution having a vector of parameters yielding the general equation for calculating Bayesian confidence bounds

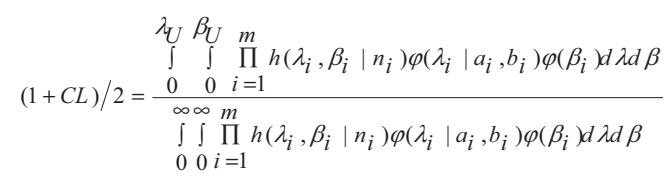

$$
(1-C L) / 2=\frac{\int_{0}^{\lambda_{L}} \int_{0} \prod_{i=1}^{m} h\left(\lambda_{i}, \beta_{i} \mid n_{i}\right) \varphi\left(\lambda_{i} \mid a_{i}, b_{i}\right) \varphi\left(\beta_{i}\right) d \lambda d \beta}{\int_{0}^{\infty} \int_{0}^{\infty} \prod_{i=1}^{m} h\left(\lambda_{i}, \beta_{i} \mid n_{i}\right) \varphi\left(\lambda_{i} \mid a_{i}, b_{i}\right) \varphi\left(\beta_{i}\right) d \lambda d \beta}
$$

The above equations can be solved to get two-sided bounds estimate for parameters.

\section{Application Example}

To illustrate the application of the proposed model, an example that incorporates many of the above aspects discussed above is outlined. At the start of development testing, some failure rate information is available from similar equipment that is already operational.

Assuming the mechanical engine will undergo multiple stages and different level tests in the development process. So, it is necessary to make a reliability growth plan in order to improve test efficiency. It should be noted that: the specifications (information, number of failures and other relevant issues) of the system studied in this paper are based on a mechanical system used in reference [19]. Here, our goal is to access a real and functional system so that we can implement the proposed model on it.

Table 1. The data of each stage [19]

\begin{tabular}{lcc}
\hline Test stage & $\begin{array}{c}\text { Number of } \\
\text { failures }\end{array}$ & Test time (hr.) \\
\hline The first stage & 26 & 90 \\
The second stage & 9 & 102 \\
The third stage & 4 & 258 \\
\hline
\end{tabular}

A reliability growth example, comprising of three stages, is shown in Table 1.As can be seen from Table 1, the first stage of the testing program is 90 hours, in which 26 failures occurred, and after the completion of the test in the first stage, it is assumed that all errors have been eliminated. In this phase, some predicted an also unpredicted weaknesses and failure modes in the design and manufacturing process are identified, and then appropriate corrective actions (or redesigns) are taken. Then the second stage of the test begins and lasts 102 hours and 9 failures are recorded in this time period. Indeed, a corrective action, or fix, for a problem failure mode in the previous typically removes a certain amount of the mode's failure intensity, but a certain amount will remain in the system. After removing the defects in the second stage, the final stage of the test is performed, and after 258 hours, the test is terminated and 4 failures are recorded in this stage.

In order to judge whether the reliability of the product is growing after the test or not, should be identified the test data follow or not the Eq. (2). For this purpose, the MTBF plot on a log-log scale with the average confidence interval/credible interval is presented in Figure 1 and shows that the product has some given value of a measure of reliability at the start of a test period and at the end of this period the value of this measure has changed hopefully, it will be improved. So, the "growth" occurred. 
As it is shown in Table 1 and Figure 1, the number of failures in each phase has been reduced and the reliability has been increased. In general, the first prototypes produced during the development of a new complex system will contain design, manufacturing, and/or engineering deficiencies. Due to these deficiencies, the initial reliability of the prototypes may be below the system's reliability goal or requirement. In order to identify and correct these deficiencies, the prototypes are often subjected to a rigorous testing program. During testing, problem areas are identified and appropriate corrective actions (or redesigns) are taken.

A reliability growth program is a well-structured process of finding reliability problems by testing, incorporating corrective actions, and monitoring the increase of the product's reliability throughout the test phases. The term "growth" is used since it is assumed that the reliability of the product will increase over time as the design changes and fixes are implemented. However, in practice, no growth or negative growth may occur. So, in real-world applications, corrective action may not be effective. But the proposed model could be representing all phases of the lifecycle (wear-out, infant mortality, useful life) of the product by using the $\beta$ parameter.

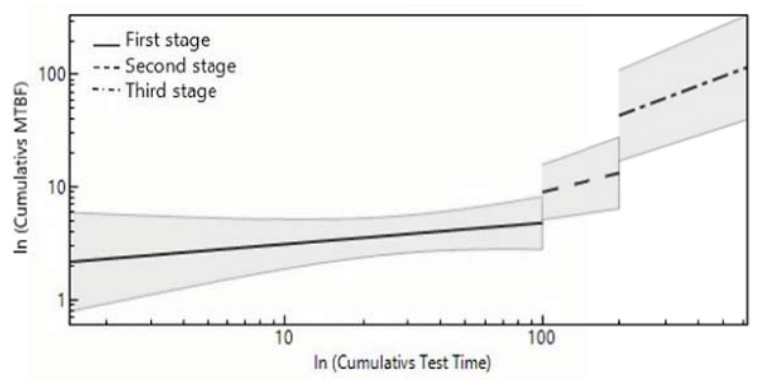

(a)

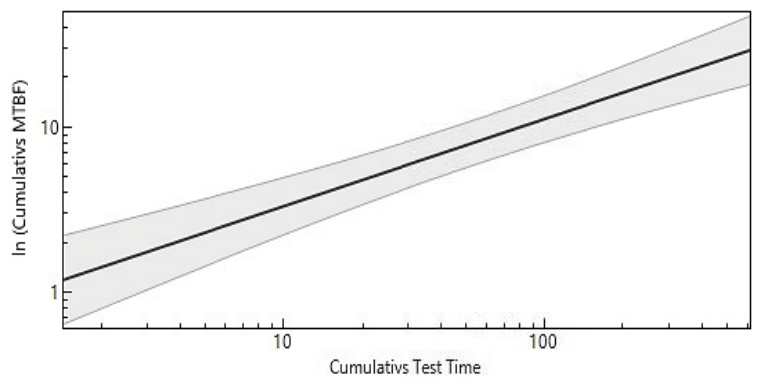

(b)

Figure 1. The reliability growth effect of different stages; (a) Bayes model, (b) Crow-AMSAA model

Table 2. Comparison of Bayes and ML estimation of $\lambda$ and $\beta$

\begin{tabular}{|c|c|c|c|c|c|c|c|c|c|c|c|c|}
\hline \multirow{3}{*}{ parameters } & \multirow{2}{*}{\multicolumn{3}{|c|}{$\begin{array}{l}\text { ML estimation } \\
\text { Crow-AMSAA }\end{array}$}} & \multicolumn{9}{|c|}{ Bayes estimation } \\
\hline & & & & \multicolumn{3}{|c|}{ The first stage } & \multicolumn{3}{|c|}{ The second stage } & \multicolumn{3}{|c|}{ The third stage } \\
\hline & mean & $\begin{array}{c}\text { Lower } \\
95 \% \\
\end{array}$ & $\begin{array}{c}\text { Upper } \\
95 \% \\
\end{array}$ & mean & $\begin{array}{l}\text { Lower } \\
\text { bond }\end{array}$ & $\begin{array}{l}\text { Upper } \\
\text { bond }\end{array}$ & mean & $\begin{array}{l}\text { Lower } \\
\text { bond }\end{array}$ & $\begin{array}{l}\text { Upper } \\
\text { bond }\end{array}$ & mean & $\begin{array}{l}\text { Lower } \\
\text { bond }\end{array}$ & $\begin{array}{l}\text { Upper } \\
\text { bond }\end{array}$ \\
\hline$\lambda$ & 2.193 & 0.843 & 5.706 & 0.617 & 0.529 & 0.853 & 0.289 & 0.153 & 0.356 & 0.378 & 0.219 & 0.518 \\
\hline$\beta$ & 0.471 & 0.355 & 0.655 & 0.812 & 0.652 & 0.912 & 0.433 & 0.253 & 0.697 & 0.134 & 0.031 & 0.312 \\
\hline
\end{tabular}

Table 3.Hyper parameters of prior distribution

\begin{tabular}{|c|c|c|}
\hline Prior Distribution & \multicolumn{2}{|c|}{ The first stage } \\
\hline \multirow{2}{*}{ Uniform } & Low & High \\
\hline & 0.770 & 0.845 \\
\hline \multirow{2}{*}{ Gamma } & Shape & Scale \\
\hline & 31.763 & 0.0208 \\
\hline
\end{tabular}

In Figure 1 the reliability growth in the test development phase has been compared in the proposed method and Crow-AMSAA model and has shown that the growth of reliability in the Crow-AMSAA model is constant in all of the stages, while in Bayesian method, the growth of reliability in each stage is changed and shown separately.

Bayes estimators of $\lambda$ and $\beta$ were compared with ML estimators, which were obtained from the CrowAMSAA model. Tables 2 give the statistical properties of the Bayes and ML estimation of the parameters $\lambda$ and $\beta$. As can be seen from Table 2 , the values of the unknown $\lambda$ and $\beta$ parameters for the Crow model with the $95 \%$ confidence interval for the whole test phase were 2.193 and 0.471 , respectively. While with the Bayesian estimation in the proposed method, these

\begin{tabular}{|c|c|c|c|}
\hline \multicolumn{2}{|c|}{ The second stage } & \multicolumn{2}{|c|}{ The third stage } \\
\hline Low & High & Low & High \\
\hline 0.405 & 0.465 & 0.105 & 0.165 \\
\hline Shape & Scale & Shape & Scale \\
\hline 20.203 & 0.0135 & 15.844 & 0.0252 \\
\hline
\end{tabular}

values are different along with the upper and lower bands at each stage of the test.

As it was mentioned before, each stage of the testing program is independent however, in the realworld system, the stage of test development is dependent. So, in this study, based on the Bayesian theory it is assumed that after phase terminating, all failures are corrected and the system is new. Therefore, this assumption leads to a lower accuracy of calculations, and it's possible the estimated parameter $\lambda$, and also $\beta$ in each phase have been deviated from the actual values. 


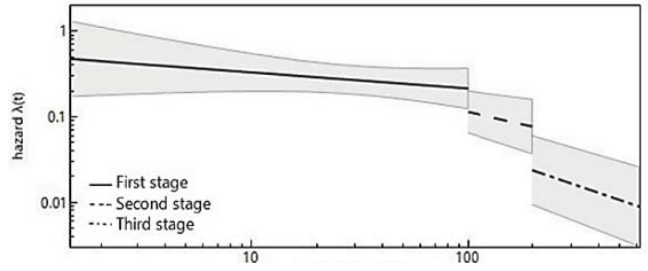

(a)

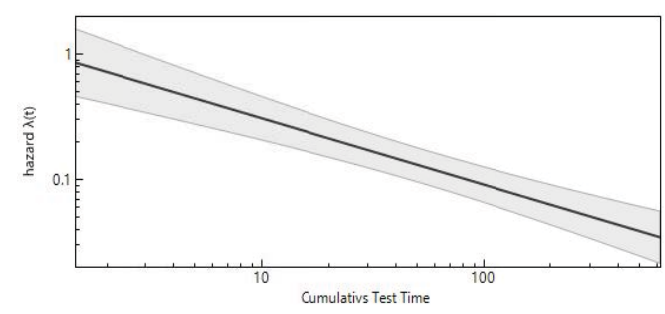

(b)

Figure 2. Hazard function; (a) Bayes model, (b) CrowAMSAA model

Figure 2 shows the hazard function versus cumulative test time on a log-log scale with the average confidence interval/credible interval based on Bayes estimation at each stage and ML estimation.

Figures 3 to 5 show the joint prior and posterior distribution of $(\lambda, \beta)$ in each of the first, second, and third stages, respectively along with the histogram, which based on 5000 iterations in Monte Carlo simulations.

As previously explained, the prior distribution of scale and growth parameter is assumed the Gamma and the Uniform distribution, respectively, and the joint prior distribution for each stage of the test is shown in Figure 3-4-5 part (a). The shape and scale parameters of the Gamma distribution and the constant value of the Uniform prior distribution for each stage are given in Table 3. Figs. 3-4-5 part (b) also shows the joint posterior distribution of $(\lambda, \beta)$ with quantiles from 0.5 to 0.990. It should be noted that in the Figs. 3-4-5 part (a), the contours of quantiles are coinciding with each other.

It is clear that the above results are obtained for a specific system in a multi-stage test process with specific conditions and will naturally be different for other systems. However, it has been shown that the efficiency of the proposed method to examine the reliability of the system is better than traditional models in multi-stage processes.

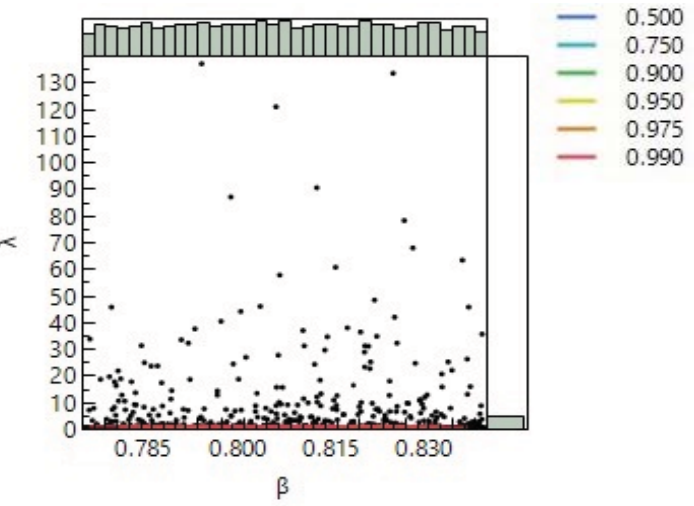

(a)

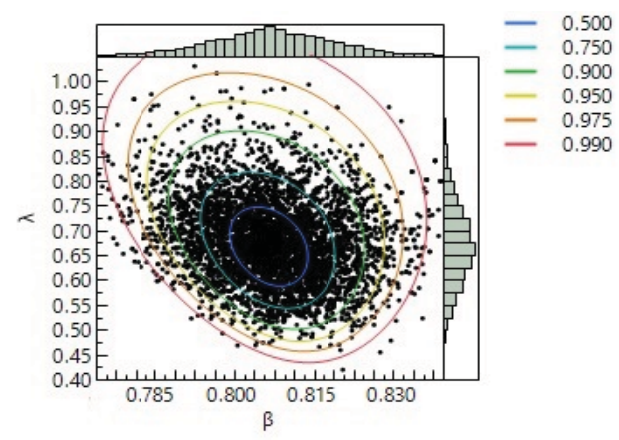

(b)

Figure 3. Contour plot of the (a) joint prior distribution \& (b) posterior distribution of $(\lambda, \beta)$ for first stage

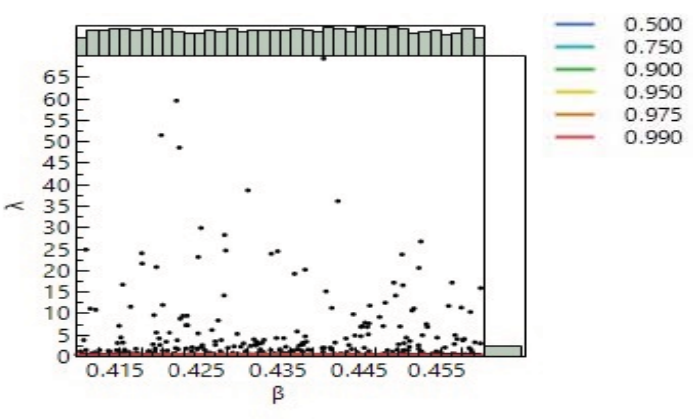

(a)

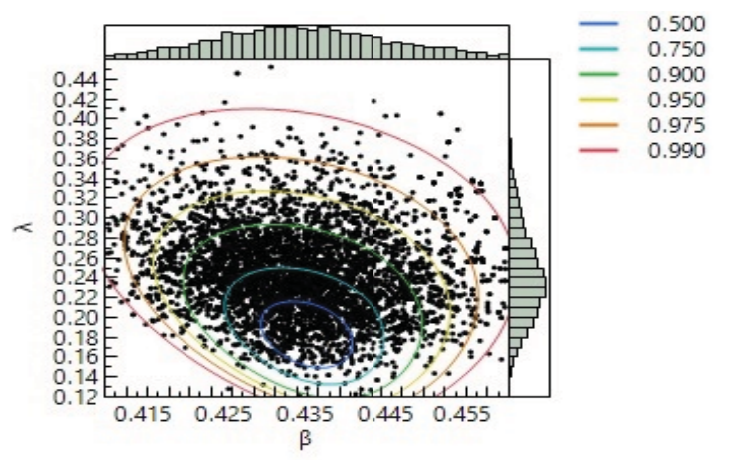

(b)

Figure 4. Contour plot of the (a) joint prior distribution \& (b) posterior distribution of $(\lambda, \beta)$ for second stage 


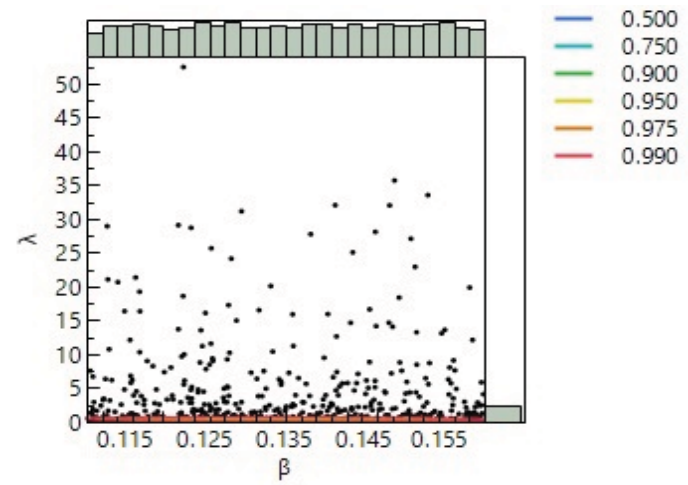

(a)

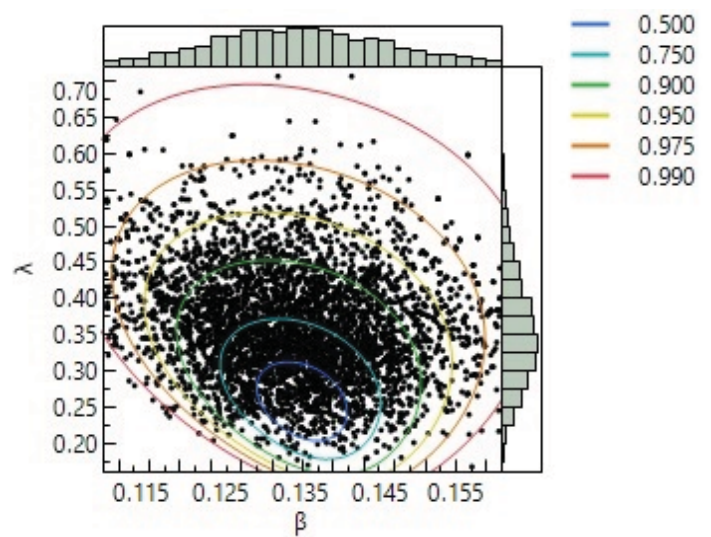

(b)

Figure 5. Contour plot of the (a) joint prior distribution \& (b) posterior distribution of $(\lambda, \beta)$ for third stage

As previously mentioned, in the proposed model, the Bayesian model is used to estimate the unknown parameters, which requires a prior distribution for the unknown model. In this study, the Gamma and Uniform distributions are used for the prior distribution, and a specific value is used for the hyper parameters of this distribution. To this purpose, reliability growth analysis and improving the reliability of various multi-stage systems using the proposed model with different sample sizes, different sampling schemes, different parameter values, and different priors are proposed for future work.

\section{Conclusions}

In this paper, the statistical analysis method of different stages of test data based on the Bayesian reliability growth model of multiple stages Weibull distribution product is investigated. By using the unique properties of the assumed prior distributions, the moments of the posterior distribution of the failure rate at various stages during a development test were obtained. Monte Carlo technique is performed to compute the approximate Bayes estimates and the corresponding credible intervals. Bayes estimates based on Gamma and Uniform priors' distribution for scale and growth unknown parameters have been compared with the corresponding ML estimates. Consequently, it was found that the results of the worked sample show the proposed method is adaptive in engineering. It was indicated that unlike traditional models such as the Crow-AMSAA model, the quality and the reliability index of products based on the proposed model is not constant and the performance of the proposed model for multiple mechanical stage systems in the development process was much better than that of the ML estimate of the traditional model.

\section{References}

[1] Duane, J., "Learning curve approach to reliability monitoring", IEEE Transactions on Aerospace, 2(2) (1964) 563-566.

[2] Crow, L.H., Reliability analysis for complex, repairable systems, Army Material Systems Analysis Activityaberdeen Poving Ground MD, 1975.

[3] Rani, R. Misra, "ML Estimates for CROW/AMSAA Reliability Growth Model for Grouped and Mixed Types of Software Failure Data", International Journal of Reliability, Quality and Safety Engineering, 11(04) (2004) 329-337.

[4] Sun, A., Kee,E., Yu,W., PopovaE., GrantomR., RichardsD., "Application of Crow-AMSAA analysis to nuclear power plant equipment performance", in: 13th International Conference on Nuclear Engineering, 2005, pp. 1-6.

[5] Barringer, H.P., "Use Crow-AMSAA reliability growth plots to forecast future system failures", (2006).

[6] Tang, Z., Zhou, W., Zhao, J., Wang, D., Zhang, L., Liu, H., Yang, Y., Zhou, C., "Comparison of the Weibull and the crow-AMSAA model in prediction of early cable joint failures", IEEE Transactions on Power Delivery, 30(6) (2015) 2410-2418.

[7] Wang, W., Xu, Y., Hou, L., "Optimal allocation of test times for reliability growth testing with interval-valued model parameters", Proceedings of the Institution of Mechanical Engineers, Part O: Journal of Risk and Reliability, 233(5) (2019) 791-802.

[8] Lee, Y., Ryu, J., Son, K., Song, S., Kim, S., Park, W., "A study on the reliability growth of multiple launch rocket system using accelerated life testing", Journal of the Korea Institute of Military Science and Technology, 22(2) (2019) 241-248.

[9] Nadjafi, M., Gholami, P., "Developing of Reliability Growth Model Based on Nonhomogeneous Poisson Process with Normal Distribution", Journal of Mechanical Engineering University of Tabriz, JMEUT-1906-2491, Accepted to Online Publish on 2020 (forthcoming).

[10] Smith, A., "A Bayesian note on reliability growth during a development testing program", IEEE Transactions on Reliability, 26(5) (1977) 346-347.

[11] Fard, N.S., Dietrich, D.L., "A Bayes reliability growth model for a development testing program", IEEE Transactions on Reliability, 36(5) (1987) 568-572. 
[12] Robinson, D., Dietrich, D., "A nonparametricBayes reliability-growth model", IEEE Transactions on Reliability, 38(5) (1989) 591-598.

[13] Lu, L., Yang, J.-p., Wang, M., “A Bayes Method for Assessing Large-Scale Electronic Equipment Reliability During External Field Test", in: Proceedings of the 6th International Asia Conference on Industrial Engineering and Management Innovation, Springer, 2016, pp. 237-244.

[14] HuangJ., KongD., CuiL., "Bayesian reliability assessment and degradation modeling with calibrations and random failure threshold", Journal of Shanghai Jiaotong University (Science), 21(4) (2016) 478-483.

[15] Ming, Z., Ling, X., Bai, X., Zong, B., "The Bayesian reliability assessment and prediction for radar system based on new Dirichlet prior distribution", in:
Journal of Physics: Conference Series, 2016, pp. 111.

[16] Ruiz, C., Pohl, E., Liao, H., Sullivan, K.M., “A Bayesian framework for accelerated reliability growth testing with multiple sources of uncertainty", Quality and Reliability Engineering International, 35(3) (2019) 837-853.

[17] Park, C., Lim, J., Lee, S., "Parameter Estimation of Reliability Growth Model with Incomplete Data Using Bayesian Method", Journal of the Korean Society for Aeronautical \& Space Sciences, 47(10) (2019) 747-752.

[18] Wang, B., Jiang, P., Li, W., Space Product Reliability Evaluation in Two-stage Development Based on Scaling Factor, IEEE Access, (2020).

[19] Proust, M., Reliability and Survival Methods, (2014). 